\title{
Dual-Resolution Active Contours Segmentation of Vickers Indentation Images with Shape Prior Initialization
}

\author{
Michael Gadermayr and Andreas Uhl* \\ Department of Computer Sciences, Salzburg University, Austria \\ uhl@cosy.sbg.ac.at
}

\begin{abstract}
Vickers microindentation imagery is segmented using the Chan-Vese level-set approach. In order to find a suitable initialization, we propose to apply a Shape-Prior gradient descent approach to a significantly resolution-reduced image. Subsequent local Hough transform leads to a very high accuracy of the overall approach.
\end{abstract}

\section{Introduction}

The Vickers hardness test uses a pyramidal diamond as indenter which is applied to a flat surface using a know force. The resulting indentation is captured using a microscope (see Fig. 1 for example images) and the diagonals are measured to determine the Vickers hardness of the material.
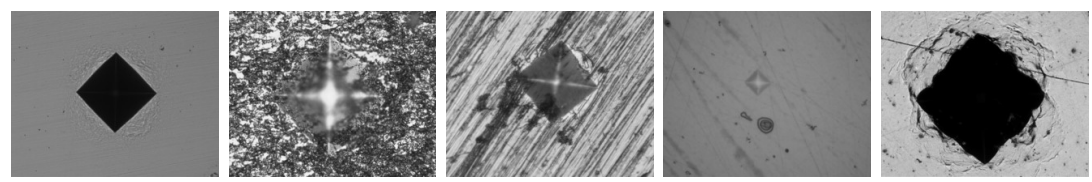

Fig. 1. Example images

There are several proposals for automated image segmentation of Vickers indentations, among them techniques based on template matching [12] and applying a local Hough transform to predefined vertex candidate regions [34]. The method introduced in [5] applies thresholding followed by a Hough transform. Other suggested methods also binarize the image using thresholding [6]7.

As can be seen in the figure, the leftmost example exhibits perfect properties for segmentation, while other images suffer from noise and/or low contrast. Therefore, Vickers indentation segmentation remains challenging. Active contours have not been investigated with respect to Vickers images so far, although the method is known to be an accurate state of the art segmentation tool. The contribution of this work is the proposal of a dual-resolution active contours algorithm (i.e. level-set approach) where the initial level set is found by a Shape-Prior gradient descent algorithm applied to a resolution-reduced image.

\footnotetext{
^ Corresponding author.
} 
This paper is structured as follows. In Sect. 2 we review existing level-set algorithms and discuss their limitations when being applied to indentation images. In Sect. 3 we introduce a Shape-Prior gradient descent method, that produces robust approximative segmentation results which serve as initialization for subsequent level-set techniques. Unlike existing Shape-Prior approaches, we restrict the evolution to the exact prior shape. In Sect.4 we fuse the components into the final algorithm: computation of an initial level set by applying the Shape-Prior gradient descent to resolution-reduced images, indentation segmentation with the highly accurate Chan-Vese region based level-set technique, and application of a local Hough transform to vertex candidate regions to optimize the accuracy of the corner detection. Section 5 compares the results to a template matching based state of the art indentation segmentation [1] with respect to accuracy and computational effort. Section 6 concludes this paper.

\section{The Level-Set Approach}

Active contours [8] are closed curves, which iteratively converge at object boundaries. The curve is forced by an energy criterion, which is based on the homogeneity of the contour on the one hand and on the image information on the other hand.

In the level-set formulation 9] an explicit parametrization by frontier points is circumvented by using an intrinsic formulation. The evolving contour is given by its level set $\Gamma$ :

$$
\Gamma=\{(x, y) \mid \phi(x, y)=0\} .
$$

$\phi(x, y)$ is a function which is 1 inside, -1 outside of the region and exactly 0 at the frontier of the evolved shape. The evolution of the frontier happens by moving the initial level set in normal direction to the contour with a specified speed $v$. There exist various different ways of calculating the speed function $v$, which influences the behavior of the evolving level set.

One quite common approach is based on the gradient information of the image 10. The speed $v$ is adjusted in order to reduce occurrences of the contour in image regions without image gradients. The edge based level-set algorithm requires the propagation of edges to increase the capture range of single edge pixels, otherwise, an exact initialization is required. Another problem is that image gradients might be weak or blurred.

To bypass these issues, a region based approach has been introduced [11. This method is based on the assumption that the object's surface and the surface outside of the object are homogeneous as far as its gray value is concerned. The following region based energy criterion has been introduced:

$$
E_{C V}=\int_{\Gamma_{\text {in }}}\left(I(v)-\overline{I_{\text {in }}}\right)^{2} d v+\int_{\Gamma_{\text {out }}}\left(I(v)-\bar{I}_{\text {out }}^{-}\right)^{2} d v+\lambda \int_{\Gamma}\|\nabla \phi(v)\| d v
$$

$I$ is the image gray value, $\bar{I}_{i n}^{-}$and $I_{\text {out }}^{-}$are the average values inside and outside of the contour, $\nabla$ is the gradient operator and $\lambda$ is the curvature weighting term. 
Intuitively, energy is low if the gray values inside the contour are equal, the gray values outside the contour are equal and the contour is smooth.

Although the region based Chan-Vese approach is known to be less vulnerable to a poor initialization, we have observed that final segmentation results are accurate only in case of close initialization. Fig. 2 illustrates the dependence of the level-set approach on the initialization. An inappropriate initialization (left rectangle) causes a wrong segmentation (crosses).
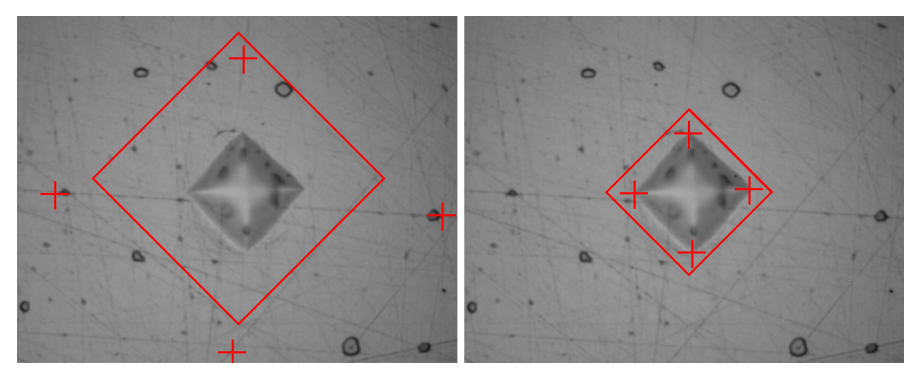

Fig. 2. The influence of the initialization (right: appropriate initialization, left: inappropriate initialization)

An additional issue is computational cost, which is tremendous in case the initialization is far away from the final contour. Having observed the importance of an appropriate initialization, we introduce a corresponding strategy in the following.

\section{The Shape-Prior Gradient Descent Method}

The approach is based on the fact, that the indentations approximately have a square shape which serves as the prior shape. Whereas traditional active contours and level-set methods evolve arbitrary curves, we consequently only evolve the parameters of a strict square template, which is represented by the following four parameters: $x_{0}$ (horizontal translation), $y_{0}$ (vertical translation), $r_{0}$ (scaling) and $\alpha$ (rotation).

The contour of the square is given by the points $(x, y)$ with the distance $d=r_{0}$ to a center $\left(x_{0}, y_{0}\right) . d$ is calculated in the following way, to ensure that the evolved contour has a square shape:

$$
\begin{aligned}
d= & \left|\left(x-x_{0}\right) \cdot \cos (\alpha)+\left(y-y_{0}\right) \cdot \sin (\alpha)\right|+ \\
& \left|\left(x-x_{0}\right) \cdot \sin (\alpha)-\left(y-y_{0}\right) \cdot \cos (\alpha)\right|
\end{aligned}
$$

Of course, this algorithm will not be able to segment Vickers images perfectly, as Vickers' shape often cannot be described by a perfect square. However, this is not our objective, but the found results can serve as good initialization for a subsequent more accurate strategy. 
The regions in- and outside of the square are given by $\Gamma_{\text {in }}$ and $\Gamma_{\text {out }}$ :

$$
\begin{gathered}
\Gamma_{\text {in }}=\left\{(x, y): d<r_{0}\right\} . \\
\Gamma_{\text {out }}=\left\{(x, y): d>r_{0}\right\} .
\end{gathered}
$$

As with the level-set approach, we define an energy criterion which is minimized by gradient descent. Different energy functions (edge based, region based) have been investigated. Tests showed that the following statistical criterion, which is derived from the approach proposed in [12, is the best choice:

$$
E=-\int_{\Gamma_{\text {in }}} \log \left(p_{\Gamma_{\text {in }}}(f(v))\right) d v-\int_{\Gamma_{\text {out }}} \log \left(p_{\Gamma_{\text {out }}}(f(v))\right) d v .
$$

$f(v)$ is an arbitrary feature of the point $v$. The higher the dimensionality of the feature vector $f$, the higher are the computational costs, as for each step of the iterative gradient descent, the $\mathrm{n}$ dimensional probability densities $p_{\Gamma_{i n}}$ and $p_{\Gamma_{\text {out }}}$ have to be calculated. Moreover, a higher dimensionality causes the empirical probability function (which is a matrix of $\mathrm{n}$ dimensions) to become a sparse matrix, as the number of matrix elements is exponentially increasing whereas the number of features stays the same. When the elements of the matrix are sparse, the empirical distribution (gathered from the pixels inside or outside the contour) cannot be utilized straightforward. Consequently, it is necessary to estimate the real probability density function which is done by applying a Gaussian Parcen window in different sizes.

Empirical tests showed, that the following feature vector produces the best results:

$$
f(v)=(I(v),\|\nabla I(v)\|) .
$$

We compute $p_{\Gamma_{\text {in }}}$ and $p_{\Gamma_{\text {out }}}$ by applying a Gaussian Parcen window to the second dimension (edge information) with variance $\sigma=2$. For the first dimension (gray value) the empirical density function is being used.

The evolved parameters are collected in the vectors $s_{i}=\left(x_{0}, y_{0}, r_{0}, \alpha\right)$. The vector $s_{0}$ is the initialization. $s_{n+1}$ is defined recursively:

$$
s_{n+1}=s_{n}+\lambda(\nabla E) .
$$

$\lambda$ which usually is a multiplicative component, is called step size. To allow lambda to act as a signum function (one pixel left, stay, one pixel right), which can deal with numerical issues, it is more generally defined as function. We use the following definition:

$$
\begin{gathered}
\lambda\left(\left(x_{1}, \ldots, x_{n}\right)^{T}\right)=\left(\operatorname{sign}\left(x_{1}\right), \ldots, \operatorname{sign}\left(x_{n}\right)\right)^{T} . \\
\nabla E=\left(\frac{d E}{d x}, \frac{d E}{d y}, \frac{d E}{d r}, \frac{d E}{d \alpha}\right)^{T} .
\end{gathered}
$$

e.g. the partial derivative of the $x$ dimension is calculated as:

$$
\frac{d E}{d x}\left((x, y, r, \alpha)^{T}\right)=\frac{1}{2} \cdot\left[E\left((x+1, y, r, \alpha)^{T}\right)-E\left((x-1, y, r, \alpha)^{T}\right)\right] .
$$


Although the introduced approach is already able to deal with local minima caused by noise, we still have not achieved a total invariance to the initialization $s_{0}$. Local minima still prevent from a proper localization of the indentation in several cases. The balloon approach [13. introduced for active contours, deals with this problem by adding an energy term, forcing the contour to become smaller or larger. Our approach allows to apply a kind of balloon force in an easy but effective way: Instead of calculating the radius $r_{0}$ by gradient descent, $r_{0}$ is simply decreased by one in each iteration of the gradient descent.

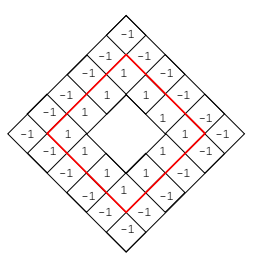

Fig. 3. Directed edge template (thickness 1) If the contour starts outside the image boundaries, it necessarily has to cross the object's boundaries, when getting smaller and smaller.

Unlike unforced gradient descent, the proposed balloon-method does not stop before $r_{0}$ becomes zero (or a defined minimum). In a second step the history of the gradient descent has to be analyzed, to get the best fitting vector $s_{\text {res }}$ from a set of several local minima. In our case the best results are achieved when using the vector $s_{i}$ with the highest response (achieved by convolution) of the image information to the template (parametrized by $s_{i}$ ) shown in Fig. 3 with a thickness of 3 pixels.

\section{Dual-Resolution Level-Set with Hough Postprocessing}

Since the main aim of the Shape-Prior gradient descent is to provide a robust initialization for subsequent more accurate techniques allowing for more adapted contours, we apply the approach to downscaled versions of the original images. This limits the computational demand of the initialization. The calculated results are upscaled and form an initial level set in the full resolution image. Subsequently, the Chan-Vese level set approach with the given initialization is conducted.

After applying the level set algorithm a decision must be made in order to identify the corners of the indentation. Unfortunately, depending on the configuration, indentations are not always segmented perfectly, but often incomplete or ragged. Even small artefacts or cut corners considerably affect the accuracy of the segmentation process if simply the topmost, the rightmost, the bottommost and the leftmost pixels of the evolved level set are identified as the corners of the indentations. Consequently, we apply the local Hough transform 34 as a post-processing strategy which approximates lines in a defined distance from the precalculated corner points of the level set technique. In the first step, the Hough transform is computed separately for each corner in a surrounding region. In the second step, the correct two lines of the Hough transform have to be selected. The first chosen line is the line with the best Hough rating and the second one is the line with the best Hough rating which is almost orthogonal to the first one. The actual corner is assumed to be the intersection of the selected two Hough lines. 


\section{Experiments}

For experimentation, 150 test images (1280x1024 pixels) acquired with EMCOTest Durascan hardware were used. In order to compare the calculated results with the ground truth, these images were manually evaluated with respect to the correct indentation vertex position by four experts independently. The ground truth was determined by taking the mean of all four measurements. For the first step of the dual-resolution approach, these images are downscaled by factor 10 (averaged with a Lanczos filter). The local Hough transform is applied in a circular area around the calculated vertex position with a radius of 60 pixels.

The results of the different strategies of the proposed dual-resolution algorithm are shown in Fig. 4. For each deviation of the calculated vertex to the ground truth in pixels as shown on the x-axis, the number of vertices with the respective distance to the real points are shown on the y-axis (bars). The rightmost bar collects the outliers (vertices with distances $\geq 20$ or $\geq 50$ pixels) while the line graph represents the relative cumulative distribution.

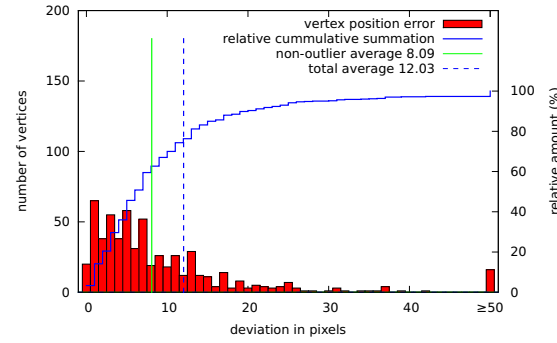

(a) Shape-Prior approach

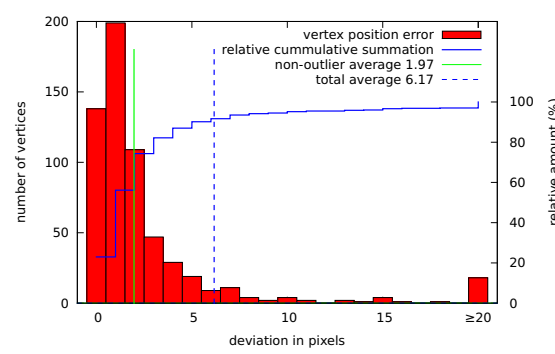

(c) Dual-resolution algorithm

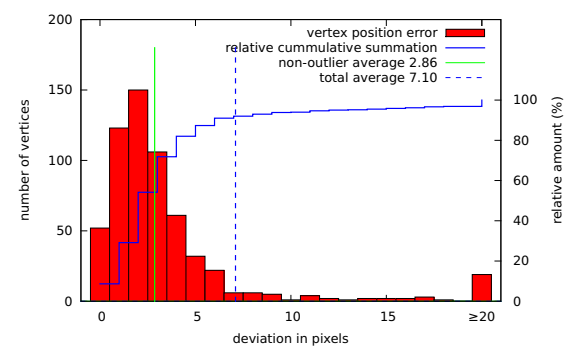

(b) Dual-resolution algorithm without local Hough transform

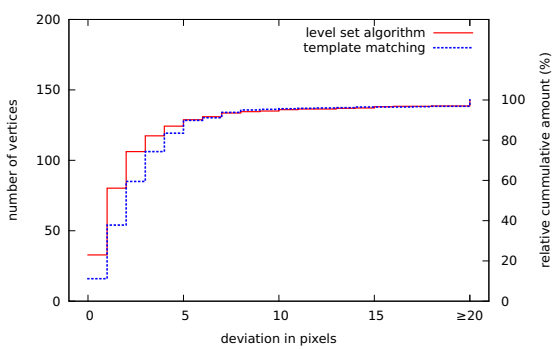

(d) Comparison to template matching

Fig. 4. Accuracy of indentation segmentation

In Fig. 4a, the provisional results (i.e. initializations for the level-set method) of the approximative Shape-Prior gradient descent approach are shown. Only vertices with a deviation of at least 50 pixels in the original image (i.e. 5 pixels in the downscaled image) are defined to be outliers. The number of outliers is already quite low, but the accuracy can be considerably increased by applying 
the level-set segmentation method as shown in Fig. 4b, For example, the ratio of vertices with a deviation of maximal 2 pixels can be increased from $21 \%$ to $53 \%$ (note that for these results, the extremal points of the segmented areas are assumed to be the indentations' vertices).

Figure 4c shows the final results achieved with the proposed dual-resolution approach plus additional local Hough transform post-processing. The accuracy is again considerably increased (e.g., $74 \%$ of the vertices have a deviation of maximal 2 pixels as compared to $53 \%$ without the local Hough transform).

We also compare the performance of the proposed dual-resolution algorithm with an alternative robust and accurate template matching approach as introduced earlier 1]. Fig. 4d compares the cumulative distribution of the proposed technique and the referenced template matching method. Especially the probability of a very exact segmentation of the indentation corner points (Euclidean distances of 0 to 5 pixels) is considerably higher with the proposed method. The number of outliers is the same.

In Fig. 5] example segmentation results of our proposed approach are shown.

Finally, we evaluate runtime performance. Shape-Prior gradient descent and the Hough transform are implemented in Java and are not optimized for execution speed. For the exact Chan-Vese segmentation stage, the high per-

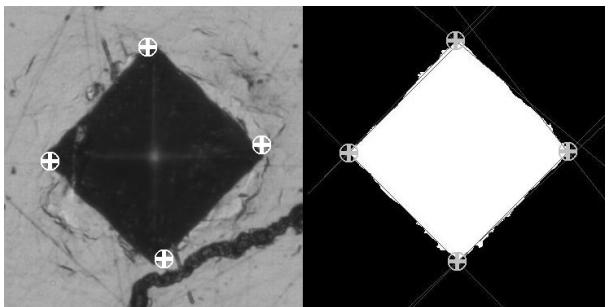

Fig. 5. Approximative results after stage 1 (left) and final results (right) formance Ofeli $\mathrm{C}++$ level-set library is used. In Table 1, average runtimes per image of the proposed method and the template matching approach [1] (implemented in Java) are shown. The tests were executed on a notebook with an Intel Core 2 Duo T5500 $1.66 \mathrm{GHz}$ processor.

Table 1. Runtime comparison

\begin{tabular}{|l|c|}
\hline Method & avg. runtime per image \\
\hline Total costs dual-resolution method & $4.3 \mathrm{~s}$ \\
\hline Proposed statistical Shape-Prior algorithm & $2.2 \mathrm{~s}$ \\
\hline Proposed region based level-set method & $1.3 \mathrm{~s}$ \\
\hline Local Hough transform & $0.7 \mathrm{~s}$ \\
\hline Referenced template matching [1] & $3.1 \mathrm{~s}$ \\
\hline
\end{tabular}

We see that although composed of three distinct procedures, the proposed technique is competitive to template matching in terms of runtime. Especially in the first stage, we still see room for runtime optimizations. 


\section{Conclusion}

The introduced dual-resolution approach consisting of a robust gradient descent localization method and an exact level-set segmentation method is highly competitive. Especially, a very exact segmentation of high quality images can be achieved.

Acknowledgment. This work has been partially supported by the Austrian Federal Ministry for Transport, Innovation and Technology (FFG Bridge 2 project no. 822682).

\section{References}

1. Gadermayr, M., Maier, A., Uhl, A.: Algorithms for microindentation measurement in automated Vickers hardness testing. In: Pinoli, J.C., Debayle, J., Gavet, Y., Cruy, F., Lambert, C. (eds.) Tenth International Conference on Quality Control for Artificial Vision (QCAV 2011). Proceedings of SPIE, vol. 8000, pp. 80000M-180000M-10. SPIE, St. Etienne (2011)

2. Maier, A., Uhl, A.: Robust automatic indentation localisation and size approximation for vickers microindentation hardness indentations. In: Proceedings of the 7th International Symposium on Image and Signal Processing (ISPA 2011), Dubrovnik, Croatia, pp. 295-300 (September 2011)

3. Ji, Y., Xu, A.: A new method for automatically measurement of vickers hardness using thick line hough transform and least square method. In: Proceedings of the 2nd International Congress on Image and Signal Processing (CISP 2009), pp. 1-4 (2009)

4. Yao, L., Fang, C.-H.: A hardness measuring method based on hough fuzzy vertex detection algorithm. IEEE Trans. on Industrial Electronics 53(3), 963-973 (2006)

5. Macedo, M., Mendes, V.B., Conci, A., Leta, F.R.: Using hough transform as an auxiliary technique for vickers hardness measurement. In: Proceedings of the 13th International Conference on Systems, Signals and Image Processing (IWSSIP 2006), pp. 287-290 (2006)

6. Mendes, V., Leta, F.: Automatic measurement of Brinell and Vickers hardness using computer vision techniques. In: Proceedings of the XVII IMEKO World Congress, Dubrovnik, Croatia, pp. 992-995 (June 2003)

7. Sugimoto, T., Kawaguchi, T.: Development of an automatic Vickers hardness testing system using image processing technology. IEEE Transactions on Industrial Electronics 44(5), 696-702 (1997)

8. Kass, M., Witkin, A., Terzopoulos, D.: Snakes: Active contour models. International Journal of Computer Vision 1(4), 321-331 (1988)

9. Osher, S., Sethian, J.A.: Fronts propagating with curvature-dependent speed: Algorithms based on hamilton-jacobi formulations. Journal of Computational Physics 79(1), 12-49 (1988)

10. Caselles, V., Kimmel, R., Sapiro, G.: Geodesic active contours. International Journal of Computer Vision 22(1), 61-79 (1997)

11. Chan, T., Vese, A.: Active contours without edges. IEEE Transactions on Image Processing 10(2), 266-277 (2001)

12. Cremers, D., Rousson, M., Deriche, R.: A review of statistical approaches to level set segmentation: integrating color, texture, motion and shape. International Journal of Computer Vision 72(2), 195-215 (2006)

13. Cohen, L.: On active contour models and balloons. CVGIP: Graphical Models and Image Processing 53(2), 211-218 (1991) 